\title{
Combined Effect of Dispersion Pressure and Concentration on Minimum Ignition Temperature of Corn Dust using Response Surface Methodology - Preliminary Investigation
}

\author{
Ushtar Arshad ${ }^{1,2}$, Azizul Buang 1,2* Khafnini Khanafiah ${ }^{2}$ \\ ${ }^{1} 1$ Centre of Advanced Process Safety (CAPS), Universiti Teknologi PETRONAS (UTP) 32610 Seri Iskandar, Perak \\ Darul Ridzuan, Malaysia \\ 2 Chemical Engineering Department, Universiti Teknologi PETRONAS (UTP) 32610 Seri Iskandar, Perak Darul \\ Ridzuan, Malaysia
}

\begin{abstract}
Corn dust is a significantly energetic dust and widely used substance in food processing industries. It not only poses dust hazards like suffocation or lungs related issues to the exposed workers but also is highly combustible under a conducive environment. In this study, the minimum ignition temperature (MIT) of corn dust clouds was evaluated at varying combinations of dispersion pressure and concentration using a Godbert-Greenwald (G-G) furnace. A response surface was generated using design expert as a tool for the application of response surface methodology (RSM). The predicted $R^{2}$ and adjusted $R^{2}$ are reasonably in close agreement as the difference is less than 0.2 . The predicted surface is found to be in good agreement with the experimental results. However, as this is a preliminary study, the model will be improved in future research.
\end{abstract}

\section{INTRODUCTION}

The handling of particulate solids is unquestionably a typical and widely used process in industries, such as pharmaceuticals, fertilisers, and the food industry. These industries largely depend on operations involving granular solids such as mixing, blending, packaging, screening, storage, size reduction, and conveying. If these granular materials are combustible, they pose a severe risk of explosion [1]. However, some materials which are almost non-flammable in bulk, also behave as explosive substances when dispersed in air as a fine powder in the presence of a sufficiently energetic source of ignition [2].

Over recent years, the augmented need for processing and storage of combustible powdered materials has led to an increased risk of dust explosions. Both the organic and inorganic dust are responsible for these explosions. Corn dust (organic) is gaining consideration being an energetic material that can cause severe damage by an explosion. It is highly combustible (fire and explosion causing agent) in nature and an essential contributor to the food industry. In particular, the crop processing industry is at a significantly higher side of risk due to the flammable nature of corn dust [3]. Seven people were killed, and 53 were severely injured in the explosion accidents occurred in a flour/feed industry in Guangdong, Henan, Zhejiang, Ningxia and Shanghai, from 2000 to 2012 China [4]. $40 \%$ of dust explosions occurred globally were related to the food industry. This proportion is alarmingly dangerous [5]. In order to avoid these explosions in the future, a thorough investigation of ignition characteristics of food dust is required.

The minimum ignition energy (MIE) and minimum ignition temperature (MIT) are the two mainstream ignition characteristic parameters. The minimum ignition temperature (MIT) is selected for the present study. The MIT is the controlling parameter of dust explosion and is crucial for evaluating the sensitivity of dust to ignition. It also plays a vital role in the design stage of powder handling equipment to make them explosion-proof.

Numerous studies have been conducted to investigate the MIT of various combustible dust. Addai et al. established the fact that the hybrid mixtures can readily ignite, owing to a lower value of MIT than that of a single powdered material. They investigated the MIT of five dust (CN4, Toner, Lycopodium, Starch and wood) with three different gases (Methane, Propane, Hydrogen) and three solvents (Ethanol, Isopropanol, Toluene). The experiments were conducted in the modified Godbert-Greenwald (G-G) furnace. The study came out with valuable observations, the MIT of gases with the addition of dust decreases even if the dust would be non-ignitable [6]. The investigations of ignition characteristics of coal have also been considered very important due to the increased accidents in coal mines. Liu et al. examined the MIT of coal and oil shale mixed dust clouds (COSMD). The investigation resulted in the fact that, with the increasing particle size of dust, MIT would also increase. The increase in MIT was

\footnotetext{
* Corresponding author: azizul.buang@utp.edu.my
} 
observed up till $50 \%$ of oil shale content and then decreased up to $80 \%$ and then finally an increasing trend above $80 \%$ was observed. The effect of inert dust (ammonium dihydrogen phosphate, sodium chloride and calcium carbonate) has also been investigated in this study and found that $80 \%$ of inert dust is enough to suppress the explosion in coal mines completely. The addition of inert dust serves the purpose of increasing the MIT of coal dust [7].

Metallic dust acquired great attention due to the explosive nature under conducive conditions of controlling parameters. Wang et al. evaluated the MIT of varying micron-sized aluminium powder. The dust exhibited an increase in MIT from $42.89 \mu \mathrm{m}-141.70 \mu \mathrm{m}$ at a constant concentration of dust cloud. Moreover, MIT touched its peaks when the concentrations were higher. The smaller particle size gives rise to the increased surface area available for combustion, enhanced absorption of oxidant, and higher rates of heat transfer also contribute to decreased MIT [8]. Manju Mittal conducted a study on MIT to observe the behaviour of micron-sized and nano-sized magnesium powder. The investigation resulted in a conclusion that nano-sized powders were more likely to explode but less violent than micron-sized powders [9].

There are multiple studies in favour of considering the explosion as a statistical phenomenon. In these investigations, considerable differences have been observed in the threshold of characteristic parameters at ostensibly same conditions. These parameters are not intrinsic properties of subject material. They may vary depending on the standard followed, testing laboratories, particle size, concentration and moisture present in the environment at the time of testing. The variation in the overlapping region of explosion and no explosion near MIE and MEC was also observed [10]. Bernard et al. investigated the probability of ignition at varying ignition energies by utilising Langlie test (statistical test). The analysis was performed on coal, aluminium, corn starch and some other dust [11].

In summary, research on combustible dust characteristic parameters, specifically the MIT of dust clouds, has mainly focused on coal, hybrid mixtures of dust and gases, metallic aluminium and magnesium powder. Scant research has examined the MIT of grain dust, specifically corn dust; in particular, the ignition as a statistical phenomenon. Therefore, in the present study, a statistical analysis was conducted to explain the effect of dispersion pressure and concentration of dust clouds in the hot zone of the $\mathrm{G}-\mathrm{G}$ furnace to predict the MIT of corn dust. The combined effects of dust concentrations and dispersion pressure on MIT were analysed by utilising RSM in Design-Expert 11.1.

\section{MATERIALS AND METHOD}

\subsection{Experimental sample}

$99.9 \%$ pure corn dust was procured and was sieved to obtain the particle size range of 125-32 $\mu \mathrm{m}$. The samples were vacuum and stored in a shaded and dry environment in anti-electrostatic packaging to avoid wetting and agglomeration. They were vacuum dried at 100 for 24 hours in a vacuum drying oven before the experiment.

\subsection{Godbert-Greenwald furnace}

Tests of the MIT were carried out in a DEKRA CHILWORTH TECHNOLOGY LTD GodbertGreenwald (G-G) furnace. The furnace is comprised of a heated vertical tube capable of reaching $1000^{\circ} \mathrm{C}$. The tube's inner diameter, height, and volume are $3.9 \times 10^{-2} \mathrm{~m}, 0.23 \mathrm{~m}$, and $2.7 \times 10^{-4} \mathrm{~m}^{3}$, respectively. Two K-thermocouples are placed near the sidewall of the tube for tube temperature adjustment and recording - a mirror located at the lower end of the furnace tube for the observation of the flame. Combustion products from the opened bottom of the furnace tube are allowed to vent into the fume cupboard.

The experiments were conducted following the International Standard ISO/IEC 80079-20-2 \& En502812-1 guidelines. First, a mass of corn dust was placed in the sample container, and the G-G furnace wall temperature and dispersion pressure were set. The sample was subsequently dispersed with compressed air as a dust cloud in the furnace tube. The combustion phenomenon was observed beyond the bottom end of the furnace tube. If the appearance of the flame was observed at the bottom of the furnace tube, the test was regarded as ignition. A schematic diagram is shown in Figure 1. It is recommended by the standard that the MIT for the dust cloud is lower than the last ignition point obtained by the experiment. The standard dictates that $10^{\circ} \mathrm{C}$ and $20^{\circ} \mathrm{C}$ will be subtracted from the last ignition point for less than $300^{\circ} \mathrm{C}$ and greater than $300^{\circ} \mathrm{C}$.

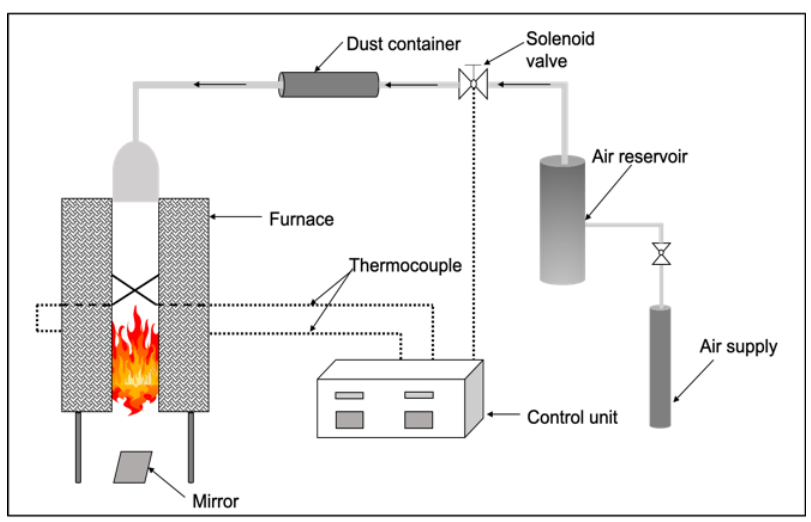

Fig. 1. Schematic of G-G Furnace.

\subsection{Response Surface Methodology (RSM)}

RSM is an appropriate methodology to study the simultaneous effects of more than one parameter on MIT along with parametric interaction. The implication of these mathematical models in real-world is the prediction capability, reducing hazards and making the process safe. A robust model with excellent predictability characteristics can foresee the hazards and 
leads toward more sustainable processes. In the current study, a model has been developed that could forecast the effects of single and multiple parameters on MIT in a comparatively broader range of operational values limited by experimental constraints. These constraints include the limited range of dispersion pressure and concentration values in this specific study.

\subsubsection{Independent variable ranges for RSM}

Stat-ease Design-Expert version 11.1 was used for the design of the experiment in the current study. The lower value of pressure was $0.1 \mathrm{barg}$, while the upper value was 0.3 barg. The range of concentration was selected from 1 to $2.6 \mathrm{~kg}$ per cubic meter $\left(\mathrm{kg} / \mathrm{m}^{3}\right)$. The lower and upper limits of the dispersion pressure and concentration lie under the applicable range provided by standard procedure. These ranges were fed into the software for the design of experiments accordingly. The actual and coded values of the selected independent parameters are shown in Table 1.

Table 1. Coded and actual values of parameters.

\begin{tabular}{|c|c|c|c|c|}
\hline Factor & Parameters & Unit & $\begin{array}{c}\text { Coded } \\
\text { Low }\end{array}$ & $\begin{array}{c}\text { Coded } \\
\text { High }\end{array}$ \\
\hline $\mathrm{A}$ & Concentration & $\mathrm{kg} / \mathrm{m}^{3}$ & $-1 \leftrightarrow 1.32$ & $+1 \leftrightarrow 2.38$ \\
\hline $\mathrm{B}$ & $\begin{array}{c}\text { Dispersion } \\
\text { pressure }\end{array}$ & $\mathrm{barg}$ & $-1 \leftrightarrow 0.13$ & $+1 \leftrightarrow 0.27$ \\
\hline
\end{tabular}

\subsubsection{RSM experimental design}

RSM removes the systematic error after estimating the experimental error while finding out the extent of the influence of control parameters on response surface MIT. RSM removes the systematic error after estimating the experimental error while finding out the extent of the influence of control parameters on response surface MIT. There are many designs such as Box-Behnken design, Hybrid design, Three-level Factorial Design and Central Composite Design (CCD) [12].

CCD was chosen as the design for this study using the design expert. The design comprised of two factors and five central points. The factors were concentration and dispersion pressure designated as $\mathrm{A}$ and $\mathrm{B}$, respectively. Accordingly, design-expert proposed 13 runs to find minimum ignition temperature achieved in the furnace at different conditions. The responses obtained from experimentation were fed into the software.

\subsubsection{RSM mathematical modelling}

Equation (1), given by design-expert, is used to determine the relationship between the response and input variables. The equation predicts the response for given levels of dispersion pressure and concentration in terms of coded factors. -1 and +1 are the low and high level of factors respectively in term coded factors by default. The selection and rejection of terms in the model are based on $95 \%$ confidence level and error probability by the design expert. The relative impacts of parameters are identified by comparing the coefficients of coded factors in the equation. The signs of coefficients depict the direct or inverse relation with response surface, a negative sign for inverse and positive sign for direct relation. The quadratic model was the best fit for these parameters. The model developed in this study had a negative coefficient of correlation for both the concentration and dispersion pressure establishing inverse relation with the MIT. This relation is in line with the fact that higher dispersion pressure causes the dust to disperse uniformly ending up in decreasing the MIT. Moreover, a higher concentration would increase heat transfer. Therefore, ignition would be favourable at lower MIT.

$$
\begin{gathered}
M I T=+431.89-7.39 * A-3.86 * B-1.52 B-4.54 A^{2} \\
-0.2046^{*} B^{2}
\end{gathered}
$$

\section{RESULTS AND DISCUSSIONS}

Corn dust was investigated using G-G furnace and the results fed into the design expert as shown in Figure 2.

\begin{tabular}{r|r|r|}
\hline $\begin{array}{c}\text { Factor 1 } \\
\text { A:Concentration } \\
\mathrm{kg} / \mathrm{m} 3\end{array}$ & $\begin{array}{c}\text { Factor 2 } \\
\text { B:Disersion pres... } \\
\text { barg }\end{array}$ & $\begin{array}{c}\text { Response 1 } \\
\text { MIT } \\
\text { Celcius }\end{array}$ \\
\hline 1.85 & 0.2 & 430 \\
\hline 1.85 & 0.2 & 435 \\
\hline 1.85 & 0.2 & 435 \\
\hline 1.11111 & 0.3 & 430 \\
\hline 2.6 & 0.111111 & 420 \\
\hline 1.1 & 0.2 & 435 \\
\hline 1.85 & 0.3 & 425 \\
\hline 2.6 & 0.3 & 405 \\
\hline 1.85 & 0.2 & 430 \\
\hline 2.6 & 0.2 & 410 \\
\hline 1.85 & 0.1 & 440 \\
\hline 1.85 & 0.2 & 430 \\
\hline 1.48148 & 0.1 & 435 \\
\hline
\end{tabular}

Fig. 2. Experimental results of MIT fed into the Design Expert.

\subsection{Statistical analysis}

In design-expert, the table given under the tab of the fit summary came out with essential statistics used to select the suitable starting point for the model. An advantageous approach of design-expert is that it selects the higher-order model when the fit summary suggests 
two models for further analysis, which is quadratic in the present study. The quadratic model was recommended to be the best fit with experimental data. The analysis of variance justified the quadratic model. This is useful in evaluating the importance of the parameters studied. ANOVA explains the main effects of dispersion pressure and concentration, their interaction effects and the error. The significant terms in this model are $\mathrm{A}, \mathrm{B}$, and $\mathrm{A}^{2}$. The F-value of 26.90 indicates that the model is significant and there is only $0.02 \%$ chance that this F-value could occur due to noise. If the p-value is less than 0.0500 then the model terms are considered significant. The software suggests that a Lack of Fit F-value 1.48, as found in this study, indicates that it is not significant relative to the pure error. Non-significant Lack of Fit is considered good by the design-expert. There is $34.66 \%$ chance, indicated by the software, that a Lack of Fit F-value could occur due to noise. ANOVA for quadratic model, given by the software that the model's accuracy for quadratic equation, is most suitable. The correlation coefficient is $\mathrm{R}^{2}=0.95$ and adjusted value is $\mathrm{R}^{2}=0.91$. However, the predicted coefficient is $\mathrm{R}^{2}=0.76$. However, the predicted $\mathrm{R}^{2}$ of 0.76 is considered to be in a reasonable agreement with the adjusted $R^{2}$ i.e. the difference is less than 0.2. An adequate precision of 17.091 indicates an adequate signal. Therefore, this model can be used to navigate the design space (Fig. 3).

\begin{tabular}{|l|r|l|r|}
\hline & \multicolumn{2}{|c|}{} & \\
\hline Std. Dev. & 3.01 & R $^{2}$ & 0.9505 \\
\hline Mean & 427.69 & Adjusted R & 0.9152 \\
\hline C.V. \% & 0.7034 & Predicted R $\mathbf{R}^{2}$ & 0.7610 \\
\hline & & Adeq Precision & 17.0910 \\
\hline
\end{tabular}

The Predicted $\mathbf{R}^{\mathbf{2}}$ of 0.7610 is in reasonable agreement with the Adjusted $\mathbf{R}^{2}$ of 0.9152 ; i.e. the difference is less than 0.2 .

Adeq Precision measures the signal to noise ratio. A ratio greater than 4 is desirable. Your ratio of 17.091 indicates an adequate signal. This model can be used to navigate the design space.

Fig. 3. Fit statistics.

\section{DIAGNOSIS BY PLOTS}

\subsection{Actual vs Predicted}

The design-expert plotted a graph between actual and predicted response based on the correlations developed by RSM, as shown in Figure 4. The significance of the quadratic model is established by the close agreement of predicted and actual values of the response.

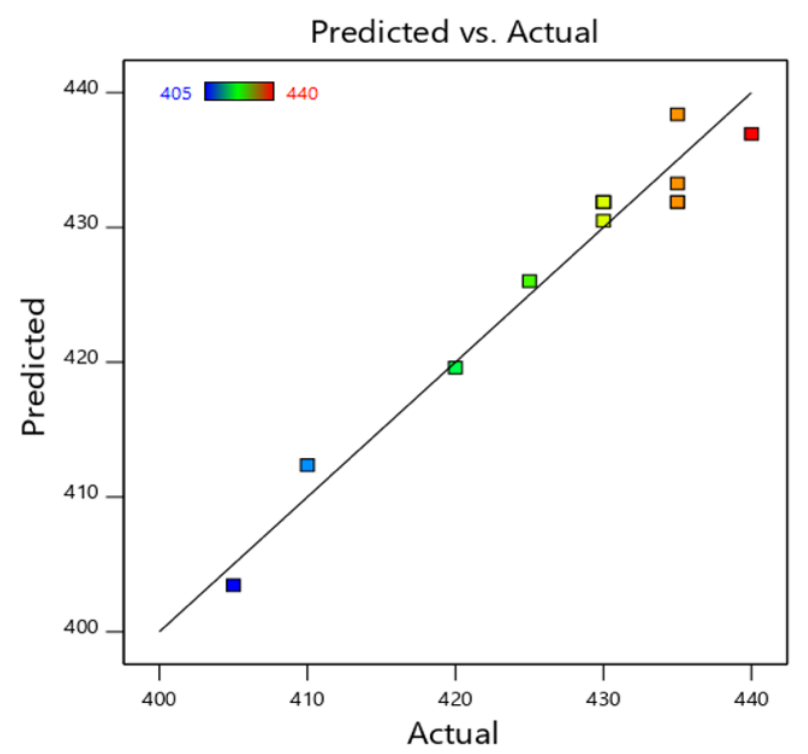

Fig. 4. Actual vs predicted plot of MIT.

\subsection{Perturbation plot}

Perturbation plot shown in figure 5. depicts the individual effect of independent variables on the response. It is highlighted in the plot by the deviation from the reference point. The effect of dispersion pressure and concentration is described in the next section below.

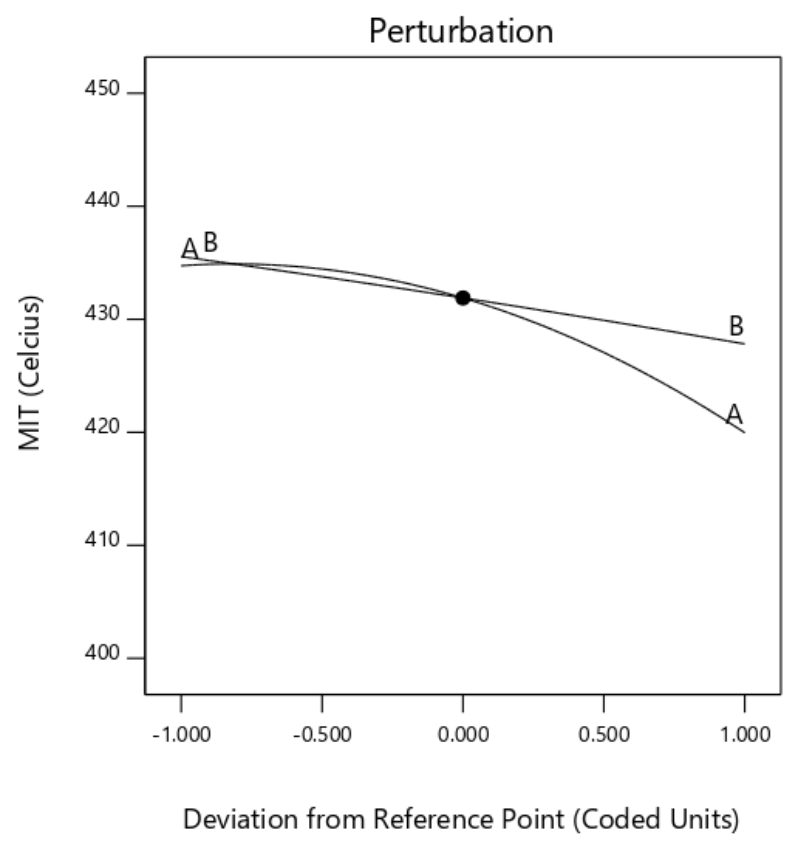

Fig. 5. Perturbation plot showing the simultaneous effects of dispersion pressure and concentration. 


\subsubsection{Effect of dispersion pressure on the response surface}

The effect of dispersion pressure was observed by varying the air pressure form (0.1-0.3 barg) by a solenoid valve in G-G furnace. It was observed that with the increasing dispersion pressure at a constant concentration, MIT was found to be decreased as illustrated in the perturbation plot in Figure 5. Dispersion pressure has an inverse relation with MIT. At higher dispersion pressure, the ignition is favoured leading to reduced MIT. This is due to the uniform dispersion of dust particles at higher dispersion pressure avoiding agglomeration. The heat conduction is more efficient with uniform dispersion, and overall with higher pressure leads to a higher amount of oxidant in the furnace [13].

\subsubsection{Effect of concentration of dust cloud on the response surface}

The concentration of dust cloud was found to have an indirect relation with the response (MIT) as indicated by the perturbation plot in Figure 5. The higher amount of ignition temperature is required for the lower concentration of the dust cloud. The rationale for this argument is that, at a lower concentration of dust, the heat released by the reaction was lower. Therefore the heat required to burn the dust comes from surrounding [13]. The concentration was obtained by the ratio of the mass of dust and volume of the furnace.

\subsection{D contour plot}

The interactive effects of concentration and dispersion pressure can be seen on the response (MIT) in the 2D contour plot. Figure 6 exhibits the interactive influence of concentration and dispersion pressure on MIT. The colour variation in the plot manifests an increase in dispersion pressure from (0.05 to 0.34$)$ bar makes the MIT lower, verifying the indirect relationship as described in section 4.2.1. Similarly, an increase in the concentration from ( 0.78 to 2.9$) \mathrm{Kg} / \mathrm{m}^{3}$ of dust in the furnace tube reduces the MIT required for ignition. Moreover, it is evident from Figure 6 that dispersion pressure has more influence on MIT in defined parameter ranges as can be seen in Figure 5 as well.

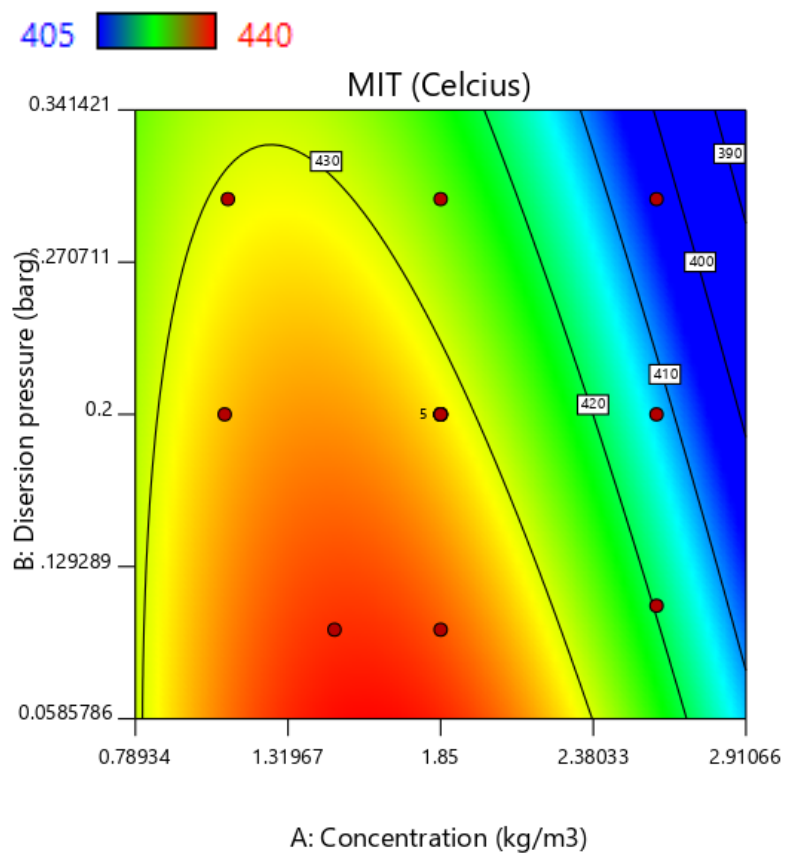

Fig. 6. $2 \mathrm{D}$ contour plot representing the effect of concentration and dispersion effect on MIT.

\subsection{D plot for effect of concentration and dispersion pressure}

A more accurate estimation of the response surface was obtained by 3D surface plot. The plot shows (Fig. 7) the influence of concentration and dispersion pressure on the MIT. In G-G furnace, when dispersion pressure is increased, proper dispersion led to the increase in the surface area available for absorption of oxidant, and hence it favoured the combustion at lower MIT [14]. An increase in the dust concentration favoured the higher rates of heat transfer to cause ignition of the dust cloud.

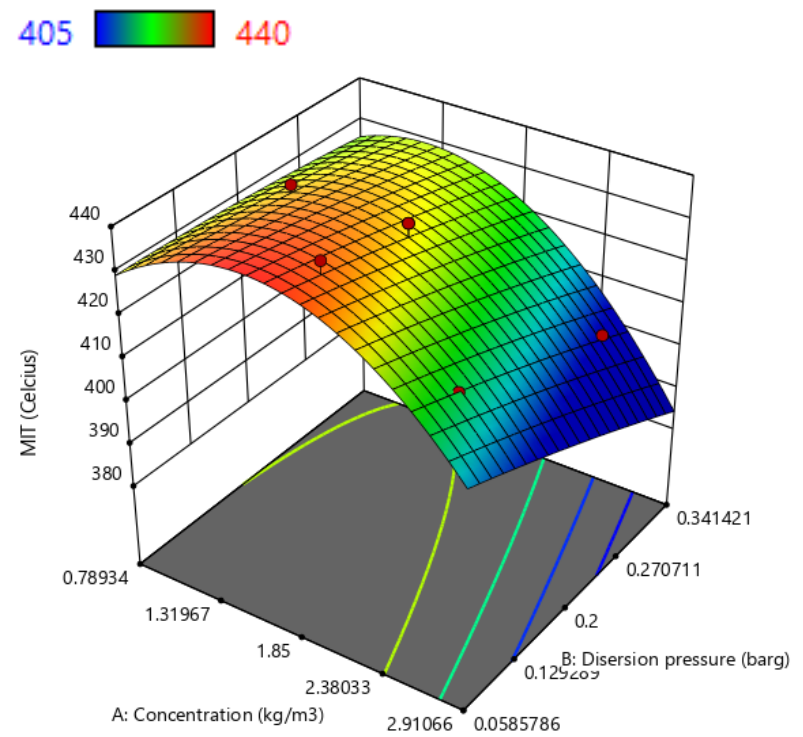

Fig. 7. 3D surface plot exhibiting the effect of concentration and dispersion pressure on MIT 


\section{CONCLUSION}

Minimum ignition temperature (MIT) of corn dust was investigated using Godbert-Greenwald furnace. This study utilizes the response surface methodology (RSM) to investigate the individual and combined interactive influences of concentration and dispersion pressure on MIT of corn dust. Fit summary in design-expert (RSM) proposed the quadratic model as the best fit for the experimental results. ANOVA justified the accessibility of the quadratic model with adjusted $\mathrm{R}^{2}=0.9152$ and predicted $\mathrm{R}^{2}=0.7610$. The predicted $\mathrm{R}^{2}$ and adjusted $\mathrm{R}^{2}$ reasonably are in agreement as the difference is less than 0.2 recommended by design-expert. The concentration of dust cloud has a significantly greater influence than dispersion pressure for corn dust with the defined range of parameters. This fact is justified by the analysis of variance, which means that by controlling the concentration, MIT can be controlled to avoid ignition and subsequent explosion. This is a preliminary study predictive modelling based on statistical analysis for corn dust. However, the model could be related to the real-time experiments to predict the MIT under different experimental conditions with limitations. In near future, there will be research on the improvement of this model to incorporate more parameters (e.g. particle size of dust, different types of dust) with a broader range of parametric variation in an extension of this research.

\section{FUTURE RECOMMENDATION}

A way forward in this line could be the use of artificial neural networking (ANN) to develop more accurate models with high predictability.

\section{References}

1. Vijayaraghavan G (2000) Review Article DUST EXPLOSIONS - A MAJOR INDUSTRIAL HAZARD.

2. Hosseinzadeh S, Berghmans J, Degreve J, Verplaetsen F (2019) A model for the minimum ignition energy of dust clouds. Process Saf Environ Prot 121:43-49

3. Zhang Q, Zhang B (2015) Effect of ignition delay on explosion parameters of corn dust/air inconfined chamber. J Loss Prev Process Ind $33: 23-28$

4. Weber M (2006) Some Safety Aspects on the Design of Sparger Systems for the. Process Saf Prog 25:326-330

5. Yuan Z, Khakzad N, Khan F, Amyotte P (2015) Dust explosions: A threat to the process industries. Process Saf Environ Prot 98:57-71

6. Addai EK, Gabel D, Krause U (2016) Experimental investigation on the minimum ignition temperature of hybrid mixtures of dusts and gases or solvents. J Hazard Mater 301:314326
7. Liu H, Chen H, Zhang X, Hu Y, Fan C (2019) Effects of different factors on the minimum ignition temperature of the mixed dust cloud of coal and oil shale. J Loss Prev Process Ind 62:103977

8. Wang Q, Fang X, Shu CM, Wang Q, Sheng Y, Jiang J, Sun Y, Sheng Z (2020) Minimum ignition temperatures and explosion characteristics of micron-sized aluminium powder. J Loss Prev Process Ind 64:104076

9. Mittal M (2014) Explosion characteristics of micron- and nano-size magnesium powders. J Loss Prev Process Ind 27:55-64

10. Zhang J, Xu P, Sun L, Zhang W, Jin J (2018) Factors influencing and a statistical method for describing dust explosion parameters: A review. J Loss Prev Process Ind 56:386-401

11. Bernard S, Lebecki K, Gillard P, Youinou L, Baudry G (2010) Statistical method for the determination of the ignition energy of dust cloud-experimental validation. J Loss Prev Process Ind 23:404-411

12. Ba D, Boyaci IH (2007) Modeling and optimization i: Usability of response surface methodology. J Food Eng 78:836-845

13. Deng J, Qu J, Wang Q, Zhai X, Xiao Y, Cheng Y, Shu CM (2019) Minimum ignition temperature of aluminium dust clouds via the Godbert-Greenwald furnace. Process Saf Environ Prot 129:176-183

14. Bagaria P, Prasad S, Sun J, Bellair R, Mashuga C (2019) Effect of particle morphology on dust minimum ignition energy. Powder Technol $355: 1-6$ 